\title{
PAPEL DA CIRURGIA BARIÁTRICA NO CONTROLE DO DIABETE MELITO TIPO II
}

\section{The role of bariatric surgery on diabetes mellitus type 2 control}

\author{
Daniella Vodola FORCINA, Bruno Olyntho de ALMEIDA, Marcelo Augusto Fontenelle RIBEIRO-Jr
}

ABCDDV/609

\begin{abstract}
Forcina DV, Almeida BO, Ribeiro-Jr MAF. Papel da cirurgia bariátrica no controle do diabete melito tipo II. ABCD Arq Bras Cir Dig 2008;21(3):130-2 RESUMO - Racional - Sabe-se que hoje um dos grandes problemas de saúde pública refere-se a diabete melito com projeção de atingir 366 milhões de pacientes até 2030, entre indivíduos insulino (DM tipo I) e não-insulino (DM tipo II) dependentes. Objetivos - Avaliar por meio de revisão ampla da literatura os resultados encontrados no controle do DM tipo II com diferentes modalidades de tratamento cirúrgico disponíveis em nosso meio. Método - A partir de 2004 foram levantados 73 trabalhos, sendo que destes 22 versavam especificamente sobre o tema nas bases de dados LiIacs, Medline, Pubmed cruzando os descritores diabete melito e cirurgia. Resultados - Dentre as operações avaliadas obtiveram resultados satisfatórios em $98,9 \%$ a derivação biliopancreática ou duodenal, em $83,7 \%$ bypass gástrico, em $71,6 \%$ gastroplastia e em $47,9 \%$ bandas gástricas. Quanto às complicações, encontram-se descritas as mais diversas, desde as da ferida operatória até casos de insuficiência hepática aguda após cirurgia bariátrica que evoluíram com necessidade de transplante ou mesmo óbito, demonstrando que a decisão pelo tratamento operatório deve ser feita de maneira criteriosa ponderando-se o custo benefício. Conclusão - As técnicas cirúrgicas bariátricas, exercem melhora do diabete tipo II e as derivações biliopancreática, duodenal switch, Scopinaro e bypass gástrico com Y de Roux são as mais indicadas.
\end{abstract}

DESCRITORES - Cirurgia bariátrica. Diabetes mellitus. Obesidade.

\section{INTRODUÇÃO}

Sabe-se que hoje um dos grandes problemas de saúde pública não apenas no Brasil, mas no mundo, refere-se a diabete melito, com projeção de atingir 366 milhões de pacientes até 2030, entre indivíduos insulino (DM tipo I) e não-insulino (DM tipo II) dependentes. Ela é responsável por mais de 2.5 milhões de mortes por ano no mundo e apresenta significante perda da expectativa de vida ${ }^{4}$.

Pacientes portadores de diabete tipo I apresentam processo degenerativo das células do pâncreas, especialmente as beta, produtoras de insulina, nesses indivíduos, há deficiência de insulina, sendo o processo fisiopatológico reação do tipo auto-imune contra as células $\beta$ pancreáticas que pode ser demonstrada por meio de marcadores genéticos. Sua maior incidência se dá entre a infância e adolescência, mas pode ocorrer também no adulto diante de situações de estresse orgânico importante ${ }^{2}$.

Dentre os fatores de risco para o DM-II destacam-se: excesso de peso; dislipidemia; hipertensão arterial; doença cardiovascular; antecedente familiar de diabete; diabete gestacional prévio ${ }^{2,3}$.

Pacientes portadores de DM II apresentam resistência à

Trabalho realizado na Universidade Cidade de São Paulo UNICID, São Paulo, SP, Brasil

Correspondência: Marcelo Augusto Fontenelle RibeiroJunior, e-mail:drmribeiro@gastrocare.med.br ação da insulina associada à incapacidade de sua secreção capaz de manter normoglicemia ${ }^{3}$.

A insulina age em vários tecidos periféricos, incluindo músculo, fígado e tecido adiposo. Seus efeitos metabólicos imediatos incluem: aumento da capitação de glicose, principalmente no tecido muscular e adiposo, aumento da síntese de proteínas, ácidos graxos e glicogênio, bem como bloqueios da produção hepática de glicose (via diminuição da neoglicogênese e glicogenólise), da lipólise e da proteólise, além disso, a insulina tem efeitos na expressão de genes e síntese protéica, assim como na proliferação e diferenciação celulares. Outras funções da insulina incluem o aumento da produção de óxido nítrico no endotélio, a prevenção da apoptose e controle da ingestão alimentar5. Com a resistência à insulina na DMII, o fígado passa a aumentar sua produção de glicose e diminuir a síntese de glicogênio e AKT; o tecido adiposo aumenta a captação de glicose, $\mathrm{CAP} / \mathrm{Cbl}$ e AKT; o tecido muscular diminui a AKT, a captação de glicose e a síntese de glicogênio; o pâncreas diminui o IRS-2 e aumenta a apoptose de células B; o hipotálamo diminui o controle da apetite e o AKT.

A etiologia ainda não estão bem estabelecidas. Há um componente genético ainda mal definido, além de obesidade, inatividade física e o envelhecimento que desencadeiam ou aceleram o aparecimento da doença. Os diabéticos tipo II têm história mais lenta, são muitas vezes assintomáticos ou diagnosticados quando da presença de 
complicações como insuficiência coronariana, neuropatia periférica, retinopatias, nefropatias, infecções ginecológicas e amputações ${ }^{1}$.

Como a associação entre pacientes diabéticos e obesos é de grande importância, foram realizados estudos relacionando as doenças a um tratamento em comum, visando a estabilização e melhora do diabete com a redução de peso. Para isso foram realizadas técnicas operatórias relacionadas à disabsorção e restrição alimentar, visando primeiramente à perda de peso, que se encontra como umas das causas principais de nível elevado de glicemia.

\section{MÉTODO}

Foram levantados 73 trabalhos desde 2004 nos bancos de dados da Medline, LILACS, e Pubmed com o escopo de identificar trabalhos apresentando diversas técnicas que estão sendo utilizadas como terapêutica cirúrgica para controle do DM tipo II como: bandas gástricas; bypass gástrico (principalmente derivação $\mathrm{Y}$ de Roux); gastroplastia (principalmente banda gastroplástica vertical); derivação biliopancreática ou duodenal com modificações e variações; bypass intestinal biliar, ileogastrectomia e bypass jejunoileal.

\section{RESULTADOS}

Na eficácia no controle do diabete, os diversos procedimentos mostraram as seguintes percentagens: derivação biliopancreática ou duodenal, 98,9\%; bypass gástrico $83,7 \%$; gastroplastia $71,6 \%$; bandas gástricas $47,9^{10,11}$. As operações mais significantes para controle da diabete foram as disabsortivas, como a derivação biliopancreática e duodenal, ou também as de mistura disabsortivas e restritivas como bypass gástrico, em contraste com as gastroplastias restritivas e bandas gástricas ${ }^{11}$. Com a bypass gástrico em Y de Roux foram observadas euglicemia e normatização da insulina dias após a realização da operação, prevenção da progressão na tolerância à glicose da DM II, posteriormente perda de $10 \%$ a $20 \%$ do peso dos pacientes ${ }^{6,13}$.

Os resultados detalhados obtidos pela revisão estão no Quadro 1.

\section{DISCUSSÃO}

A relação entre as alterações metabólicas dos pacientes e a perda de peso sugere interação entre a quantidade de tecido adiposo e o nível glicêmico, tendo os fatores endócrinos como principais reguladores desse sistema ${ }^{2}$.

Como destaque, tem-se a leptina, um derivado da adiponectina, mediada por feedback entre o tecido adiposo e o hipotálamo. A grelina, responsável pela fome, situada no estômago e os hormônios PYY3 e GLP-1 que são responsáveis pela saciedade, encontram-se no final do intestino, o que reforça a idéia de que as operações disabsortivas ou mistas apresentem melhor resultado quando comparadas às exclusivamente restritivas ${ }^{6,7,9}$.

A glicotoxicidade caracteriza-se por efeitos adversos da hiperglicemia crônica sobre a função da célula beta e incluem três consequências distintas: diminuição da tolerância à glicose; exaustão das células beta e redução da massa de células beta por apoptose. A diminuição da tolerância à glicose deve-se à refratariedade reversível do mecanismo de liberação da insulina produzida após a exposição a níveis elevados de glicemia devida a autooxidação da célula beta. Nessas circunstâncias, ocorre mecanismo fisiológico adaptativo para preservar a célula beta, reduzindo a primeira fase de produção de insulina

\begin{tabular}{|c|c|c|c|c|c|c|c|}
\hline AUTORES & $\mathbf{N}$ & OPERAÇÃO & $\downarrow$ PESO & PRÉ OPERATÓRIO & PÓS OPERATÓRIO & RESOLUÇÃO & $\downarrow$ MEDICAMENTO \\
\hline \multirow[t]{3}{*}{ Pinkney et al. } & $\mathrm{n}=186$ & GBP & $33 \%$ & Insulina $=44$ & Insulina $=14$ & $83 \%$ & $\mathrm{~N} / \mathrm{C}$ \\
\hline & $\mathrm{n}=70$ & LGB & $27 \mathrm{~kg}$ & Insulina $=4 / \mathrm{OHA}=29$ & Insulina $=4 / \mathrm{OHA}=8$ & $70 \%$ & $\mathrm{~N} / \mathrm{C}$ \\
\hline & $\mathrm{n}=24$ & VBG & $30 \mathrm{~kg}$ & Insulina $=7 / \mathrm{OHA}=22$ & Insulina $=4 / \mathrm{OHA}=6$ & $\mathrm{~N} / \mathrm{C}$ & $\mathrm{N} / \mathrm{C}$ \\
\hline Rubino et al. & $\mathrm{n}=10$ & LGB & $\mathrm{N} / \mathrm{C}$ & Insulina/OHA $=6$ & Insulina/OHA $=0$ & $100 \%$ & $0 \%$ \\
\hline \multirow[t]{2}{*}{ Patriti et al. } & $\mathrm{n}=478$ & RYGBP & $\mathrm{N} / \mathrm{C}$ & $\mathrm{OHA}=478$ & $\mathrm{OHA}=88$ & $81 \%$ & $12 \%$ \\
\hline & $\mathrm{n}=150$ & BPD & $\mathrm{N} / \mathrm{C}$ & $\mathrm{OHA}=150$ & $\mathrm{OHA}=1$ & $99.3 \%$ & $0.7 \%$ \\
\hline \multirow[t]{4}{*}{ Geloneze et al. } & $\mathrm{N} / \mathrm{C}$ & LGB & $47,5 \%$ & $\mathrm{~N} / \mathrm{C}$ & $\mathrm{N} / \mathrm{C}$ & $\mathrm{N} / \mathrm{C}$ & $\mathrm{N} / \mathrm{C}$ \\
\hline & $\mathrm{N} / \mathrm{C}$ & GBP & $61,6 \%$ & $\mathrm{~N} / \mathrm{C}$ & $\mathrm{N} / \mathrm{C}$ & $71,6 \%$ & $\mathrm{~N} / \mathrm{C}$ \\
\hline & $\mathrm{N} / \mathrm{C}$ & VBG & $68,2 \%$ & $\mathrm{~N} / \mathrm{C}$ & $\mathrm{N} / \mathrm{C}$ & $47,9 \%$ & $\mathrm{~N} / \mathrm{C}$ \\
\hline & $\mathrm{N} / \mathrm{C}$ & BPD & $70,1 \%$ & $\mathrm{~N} / \mathrm{C}$ & $\mathrm{N} / \mathrm{C}$ & $83,7 \%$ & $\mathrm{~N} / \mathrm{C}$ \\
\hline Guidone et al. & $\mathrm{n}=10$ & BPD & $10 \%(4 \mathrm{sem})$ & Insulina $=10$ & Insulina $=0$ & $100 \%$ & $\mathrm{~N} / \mathrm{C}$ \\
\hline \multirow[t]{3}{*}{ Mango et al. } & $\mathrm{N} / \mathrm{C}$ & GBP & $38 \%$ & $\mathrm{~N} / \mathrm{C}$ & $\mathrm{N} / \mathrm{C}$ & $\mathrm{N} / \mathrm{C}$ & $\mathrm{N} / \mathrm{C}$ \\
\hline & $\mathrm{N} / \mathrm{C}$ & VBG & $26 \%$ & $\mathrm{~N} / \mathrm{C}$ & $\mathrm{N} / \mathrm{C}$ & $\mathrm{N} / \mathrm{C}$ & $\mathrm{N} / \mathrm{C}$ \\
\hline & $\mathrm{N} / \mathrm{C}$ & LGB & $21 \%$ & $\mathrm{~N} / \mathrm{C}$ & $\mathrm{N} / \mathrm{C}$ & $\mathrm{N} / \mathrm{C}$ & $\mathrm{N} / \mathrm{C}$ \\
\hline \multirow[t]{2}{*}{ Alexandrides et al. } & $\mathrm{n}=26$ & RYGBP & $30 \%$ & $\mathrm{~N} / \mathrm{C}$ & $\mathrm{N} / \mathrm{C}$ & $88.5 \%$ & $11,5 \%$ \\
\hline & $\mathrm{n}=111$ & BPD-RYGBP & $33 \%$ & $\mathrm{~N} / \mathrm{C}$ & $\mathrm{N} / \mathrm{C}$ & $99.1 \%$ & $0.9 \%$ \\
\hline Scopinaro et al. & $\mathrm{n}=7$ & BPD & $18 \%$ & $\mathrm{~N} / \mathrm{C}$ & $\mathrm{N} / \mathrm{C}$ & $100 \%$ & $\mathrm{~N} / \mathrm{C}$ \\
\hline Halpern et al. & $\mathrm{n}=39$ & II-SG / II-DSG & $20 \%$ & Insulina $=3 / \mathrm{OHA}=22$ & $\mathrm{~N} / \mathrm{C}$ & $47.4 \%$ & $52.6 \%$ \\
\hline \multirow[t]{3}{*}{ Parikh et al. } & $\mathrm{n}=218$ & LGB & $\mathrm{N} / \mathrm{C}$ & Insulina $=18 \% / \mathrm{OHA}=83 \%$ & Insulina $=18 \%$ / OHA $34 \%$ & $\mathrm{~N} / \mathrm{C}$ & $\mathrm{N} / \mathrm{C}$ \\
\hline & $\mathrm{n}=53$ & RYGB & $\mathrm{N} / \mathrm{C}$ & Insulina $=28 \% / \mathrm{OHA}=87 \%$ & Insulina $=13 \% / \mathrm{OHA}=13 \%$ & $\mathrm{~N} / \mathrm{C}$ & $\mathrm{N} / \mathrm{C}$ \\
\hline & $\mathrm{n}=11$ & $\mathrm{BPD} / \mathrm{DS}$ & $\mathrm{N} / \mathrm{C}$ & Insulina $=82 \% / \mathrm{OHA}=82 \%$ & Insulina $=13 \% / \mathrm{OHA}=13 \%$ & $\mathrm{~N} / \mathrm{C}$ & $\mathrm{N} / \mathrm{C}$ \\
\hline
\end{tabular}

GBP - bypass gástrico; LGB - bypass gástrico por laparoscopia; VGB - gastroplastia vertical com banda; RYGBP - banda gástrica com Y de Roux; BPD - derivação biliopancreática; II-SG/DSG - Interposição ileal com gastrectomia; BPD/DS - derivação biliopancreática com ou sem derivação duodenal; OHA - hipoglicemiante oral, N/C - não consta.

QUADRO 1 - Resultados obtidos por diversos autores com diferentes operações 
e promovendo menor supressão da liberação hepática de glicose após as refeições, aumentando ainda mais a hiperglicemia pós-prandial ${ }^{6}$. A conseqüência prática direta da glicotoxicidade é a incapacidade de alguns pacientes com glicemia bastante elevada, geralmente acima de $300 \mathrm{mg} / \mathrm{dL}$ em jejum, no sentido de não conseguirem redução adequada dos níveis glicêmicos apenas com o tratamento oral, necessitando de um período variável de terapia insulínica para restaura-los em patamares aceitáveis. Para muitos pacientes, essa conduta terapêutica controla a glicotoxicidade e permite que o paciente passe a responder adequadamente aos antidiabéticos orais 9 .

A lipotoxidade geralmente ocorre em portadores de DMII e obesidade, com adiposidade visceral. Neste caso, são os níveis elevados de ácidos graxos, por períodos prolongados, que resultam em resposta diminuída das células beta aos níveis de glicose sanguínea. Em condições normais, os ácidos graxos são uma forma de energia para as células beta, mas se tornam tóxicos quando em concentrações cronicamente elevadas e em indivíduos geneticamente predispostos ao DMII ${ }^{9}$. Os efeitos deletérios dos ácidos graxos são mediados pela presença do excesso de glicose, uma vez que os lípides aumentados não alteram a função das células beta em modelos animais mantidos em níveis normais de glicemia principalmente quando há inadequação ou falha do tratamento levando a descontrole do nível glicêmico sanguíneo
O tratamento na DMII pode ser realizado com uso de hipoglicemiantes orais, insulina coadjuvante a exercícios físicos e dieta adequada. Cabe somente ao médico indicar, prescrever e orientar o paciente em seu correto tratamento

A relação da melhoria do diabete com a cirurgia bariátrica deve-se à melhora da tolerância à glicose, ocasionada primeiramente pela redução de peso devida menor ingestão calórica9. Acredita-se que a relação com a perda de peso deve-se à diminuição do tecido adiposo, acarretando diminuição da produção e atuação de várias substâncias produzidas pelos adipócitos, e entre elas estão: angiotensinogenio, PAI-1, IL-6, resistina, adiponectina, leptina, TNF-alfa entre outras4.

Muitos pacientes após a operação saem do hospital até mesmo sem o uso da insulina, e com alguma perda do peso excessivo, calculada pela fórmula: peso perdido/ excesso de peso (total do peso pré-operatório - peso ideal) X 100. A variação dessa porcentagem dependerá da evolução e técnica operatória utilizada4.

\section{CONCLUSÃO}

As técnicas cirúrgicas bariátricas exercem melhora do diabete tipo II e as derivações biliopancreática, duodenal switch, Scopinaro e bypass gástrico com Y de Roux são as mais indicadas.

Forcina DV, Almeida BO, Ribeiro-Jr MAF. The role of bariatric surgery on diabetes mellitus type 2 control. ABCD Arq Bras Cir Dig 2008;21(3):130-2

ABSTRACT - Introduction - One of the today's biggest health problems is diabetes mellitus and is projected to have 366 million patients in 2030 , between insulin (DM I) and non-insulin dependent (DM II). Aim - To evaluate through a literature review the surgical results in the type II using surgical methods available in bariatric surgery. Method - The scientific databases Lilacs, Medline and Pubmed were used to collect papers with the surgical treatment of diabetes and 73 articles were found, being 22 specifically related to the subject. Results - Surgery shows inicial acceptable results with best ones related to biliopancreatic diversion in $98,9 \%$ of cases, gastric bypass $83,7 \%$, gastroplasty $71,6 \%$, and gastric banding $47,9 \%$. There are mentioned many complications some of them progressing to death and, so, surgical treatment must be done with responsibility and analyzing cost-benefit in each procedure. Conclusion - Bariatric surgery gives improvement in type II diabetes and biliopancreatic diversion and Roux-em-Y gastric bypass are the most indicated to this type.

HEADINGS - Bariatric surgery. Diabetes mellitus. Obesity.

\section{REFERÊNCIAS}

1 Andreollo NA, Michelino MU, Brandalise NA, Lopes LR, Trevisan MA, 1. Adams TD, Gress RE, Smith SC, Halverson RC, Simper SC, Rosamond WD, LaMonte MJ, Stroup AM, Hunt SC. Long-Term Mortality after Gastric Bypass Surgery. N Engl J Med. 2007;357:753-61.

2. Alexandrides TK, Skroubis G, Kalfarentzos F. Resolution of Diabetes mellitus and Metabolic Syndrome following Roux-en-Y Gastric Bypass and a Variant of Biliopancreatic Diversion in Patiens with Morbid Obesity. Obes Surg. 2007; 17:176-84

3. Anvari M. Is type 2 diabetes a surgical disease? Can J Surg. 2007;50:249-50.

4. Buchwald H, Avidor Y, Braunwald E, Jensen MD, Pories W, Fahrbach K, Schoelles K. Bariatric Surgery: A Systematic Review and Meta-Analysis. JAMA. 2004:292:1724-37.

5. DePaula AL, Macedo ALV, Rassi N, Machado CA, Schraibman V, Silva LQ, Halpern A. Laparoscopic treatment of type 2 diabetes mellitus for patients with a body mass index less than 35 . Surg Endosc. 2007;22:706-16.

6. Geloneze B, Pareja JC. Cirurgia Bariatrica Cura a Síndrome Metabólica? Arq Bras Endocrinol Metabol. 2006;50:400-5.
7. Guidone C, Manco M, Valera-Mora E, Iaconelli A, Gniuli D, Mari A, Nanni G, Castagneto M, Calvani M, Mingrone G. Mechanisms of Recovery From Type 2 Diabetes After Malabsorptive Bariatric Surgery. Diabetes. 2006;55:2025-31.

8. Pinkney J, Kerrigan D. Current status of bariatric surgery in the treatment of type 2 diabetes. Obes Rev. 2004;5:69-78.

9. Rubino F, Gagner M, Gentileschi P, Kini S, Fukuyama S, Feng J, Diamond E. The Early Effect of the Roux-en-Y Gastric Bypass on Hormones Involved in Body Weight Regulation and Glucose Metabolism. Ann Surg. 2004;240:236-42.

10. Rubino F. Bariatric surgery: effects on glucose homeostasis. Curr Opin Clin Nutr Metab Care. 2006;9:497-507.

Fonte de financiamento: não há Conflito de interesse: não há Recebido para publicação: 15/03/2008 Aceito para publicação: 21/06/2008 